\title{
Application of the variational calculus to wetting phenomena in chemical engineering
}

\author{
F. P.-A. Cortat S. J. Miklavcic*
}

(Received 16 March 2006; revised 15 April 2007)

\begin{abstract}
The problem of determining the equilibrium shape of a wetting meniscus is proposed as a rich example of application of the variational method. The problem describes a common-place phenomena that is conceptually simple and physically tangible for undergraduate students. The proposal has the further appeal in that it illustrates how more abstract variational boundary equations can be implemented. It also represents a system that gives rise to both stable (minimum) and unstable (maximum) wetting profiles, and by utilizing these it leads to a criterion earmarking eventual existence limits of solutions of the variational equations.
\end{abstract}

* Department of Science and Technology, Linköping University, Campus Norrköping, S-601 74, Norrköping, Sweden. mailto:freco@itn.liu.se

See http://anziamj .austms.org.au/V47EMAC2005/Cortat2 for this article, (c) Austral. Mathematical Soc. 2007. Published July 20, 2007. ISSN 1446-8735 


\section{Introduction}

One of the most fundamental principles of physics requires that a system, when faced with a choice between several possible states, select the energetically most favorable option, the state with the lowest energy. An enormous variety of phenomena are governed by this principle, and a range of mathematical methods have been developed to determine the minimal possible energy values. Among these the variational minimization technique is one of the most common.

Determining the minima of the energy surface as a function of scalar variables can be achieved by use of standard derivative methods. However, physical phenomena are often governed by energies that are functionals, in which cases the minima are determined by a generalization of the scalar methods, referred to as variational minimization of functionals. The procedure is based on assuming the existence of a function or functions for which the functional is an extremum and perturbing this function or functions requiring that terms of first order in the perturbation vanish $[1,2]$. This generally delivers a system of differential equations, the Euler-Lagrange equations, whose solution guarantees an extremum of the energy relative to all neighboring functions. One slight drawback with this idea, both in the scalar and energy functional cases, is that it results in equations leading to extrema of the quantity to be minimized, but not directly to an energy minimum.

Nonetheless, in a standard physics or applied mathematics curriculum, the subject of variational calculus is considered an essential tool in a physicist's or applied mathematician's theoretical hardware. However, although the common student course on the variational method, based on standard texts in mechanics [3,4] or mathematical methods of physics $[1,2,5]$ or even applied mathematics $[6,7]$, covers the theoretical basis of the variational calculus, only elementary examples are taken up to illustrate the principles. The classic example is a hanging cable with fixed endpoints. Other examples often feature linear systems, again with simple boundary conditions, that 
generally lead to a single unique solution, automatically guaranteed to be a minimum. With such examples the full scope of the method remains unexposed. Moreover, the more difficult aspect of nontrivial boundary conditions is not always addressed, and certainly cannot be explored in detail with simple examples. Indeed, we argue that these nontrivial boundary conditions are not often taken up because of the absence of a suitable illustrative example.

In this article we propose a more involved physics problem to illustrate the variational calculus. This rich example we consider can add considerably to student understanding and appreciation of the method. The problem, arising in many areas of chemical engineering systems $[8,9]$, involves determining the equilibrium shape of a wetting meniscus formed around an axisymmetric solid object that is partially immersed in an infinite liquid bath. The appeal of the present example problem is four-fold. Firstly, it addresses a conceptually simple, physically intuitive system that can be easily grasped by the physics student, thus diminishing the educational burden. Secondly, the special feature that the equilibrium fluid interface - wetting meniscus - must be in contact with the solid leads naturally to use of the nontrivial, so-called natural boundary conditions [2], otherwise considered abstract generalizations of the method. Thirdly, the system of differential equations resulting from a functional variation can possess at least two plausible solutions, one stable (minimum) and one unstable (saddle point or maximum). Thus, the educator demonstrates one important practical aspect of the variational method: that the Euler-Lagrange system is a necessary but not sufficient system leading to the minimizing solution. Finally, the two solutions in combination lead to a feature seldom addressed in teaching the method: the possibility that the variational method fails altogether to give a minimizing solution. Inherent in the wetting problem is an absolute stability or existence limit that is possible to track numerically. 


\section{The variational method}

This section introduces the problem of determining the equilibrium shape of a wetting meniscus via the variational method. Since the overall aim of this article is to present a sufficiently simple problem to exemplify the more intricate features of the variational method, we shall restrict the example to its most basic form. More complicated models that deal with wetting and attempt to incorporate molecular and other details exist [10], but these are not discussed here.

Three surface areas are featured: a solid-gas contact area, a solid-liquid contact area, $A_{\Xi}$, and a liquid-gas contact area, $A_{\Omega}$, each with an intrinsic surface energy density denoted, respectively, by $\gamma_{S G}, \gamma_{S L}$ and $\gamma$. These, together with the densities of the liquid, $\rho_{L}$, and gas, $\rho_{G}$, are the only material parameters determining the physical shape of the meniscus profile. In terms of geometry, we assume that the wetted solid is cylindrically symmetric, and that the fluids extend infinitely away from the vicinity of the solid. Thus, we do not address the issue of volume constraints.

The remainder of this section is divided into three subsections. The first deals with an alternate derivation of the shape equation, not uncommon in chemical engineering texts. The second subsection introduces the variational method for the relatively simple problem of wetting of an infinite cylinder, typically addressed in chemical engineering texts, and reproduces the equation derived in the first subsection. On the other hand, the third subsection deals with wetting of the paraboloid cylinder and represents a less trivial application of the variational theory.

\subsection{Laplace and Young-Dupre equations of capillarity}

One recognized way of determining the equilibrium shape of the interface between two stationary fluids involved in the wetting of a solid surface is 
by using a force balance argument [11]. A curved interface in equilibrium requires that the forces acting on a surface element be in balance. In the case in point this force balance is expressed in terms of the local mean (Gaussian) curvature and the pressure difference across the ideal surface:

$$
\gamma\left(\frac{1}{R_{1}}+\frac{1}{R_{2}}\right)=\Delta p
$$

Here, the pressure difference comes from buoyancy effects, $g \Delta \rho=g\left(\rho_{L}-\rho_{G}\right)$. The two principal radii of curvature, $R_{1}$ and $R_{2}$, are expressed differentially in terms of the local shape function, $z=z(r)$. By doing so the usual Laplace differential equation governing the shape of the profile [11] is obtained,

$$
\gamma\left[\frac{z_{r r}}{\left(1+z_{r}^{2}\right)^{3 / 2}}+\frac{z_{r}}{r \sqrt{1+z_{r}^{2}}}\right]=g \Delta \rho z .
$$

A force balance argument, applied to the line of three phase contact on the solid surface, is traditionally used to deduce a boundary condition to invoke when (1) is integrated: the vector sum of the three surface tensions acting on the contact line is zero. Applying the reasoning to the component of the net force in the direction parallel to the solid (Figure 1b), leads to the well-known force balance

$$
\gamma \cos \theta_{0}=\gamma_{S G}-\gamma_{S L}=: \Delta \gamma
$$

This relation is known as the Young-Dupre equation for the contact angle $\theta_{0}=\phi_{0}+\pi / 2$, defined as the angle between the fluid interface and the solid surface, measured through the liquid region. $\phi_{0}$ denotes the angle the fluid interface makes with the horizontal (see Figure 1). Relation (2) depends only on the different surface tension coefficients and is independent of either the curvature of the solid, the curvature of the contact line or the gravitational properties of the system. In the force-balance derivation of (2), one implicitly assumes the contact line has zero curvature.

Equation (2) can be rewritten in terms of the slope of the profile at the 

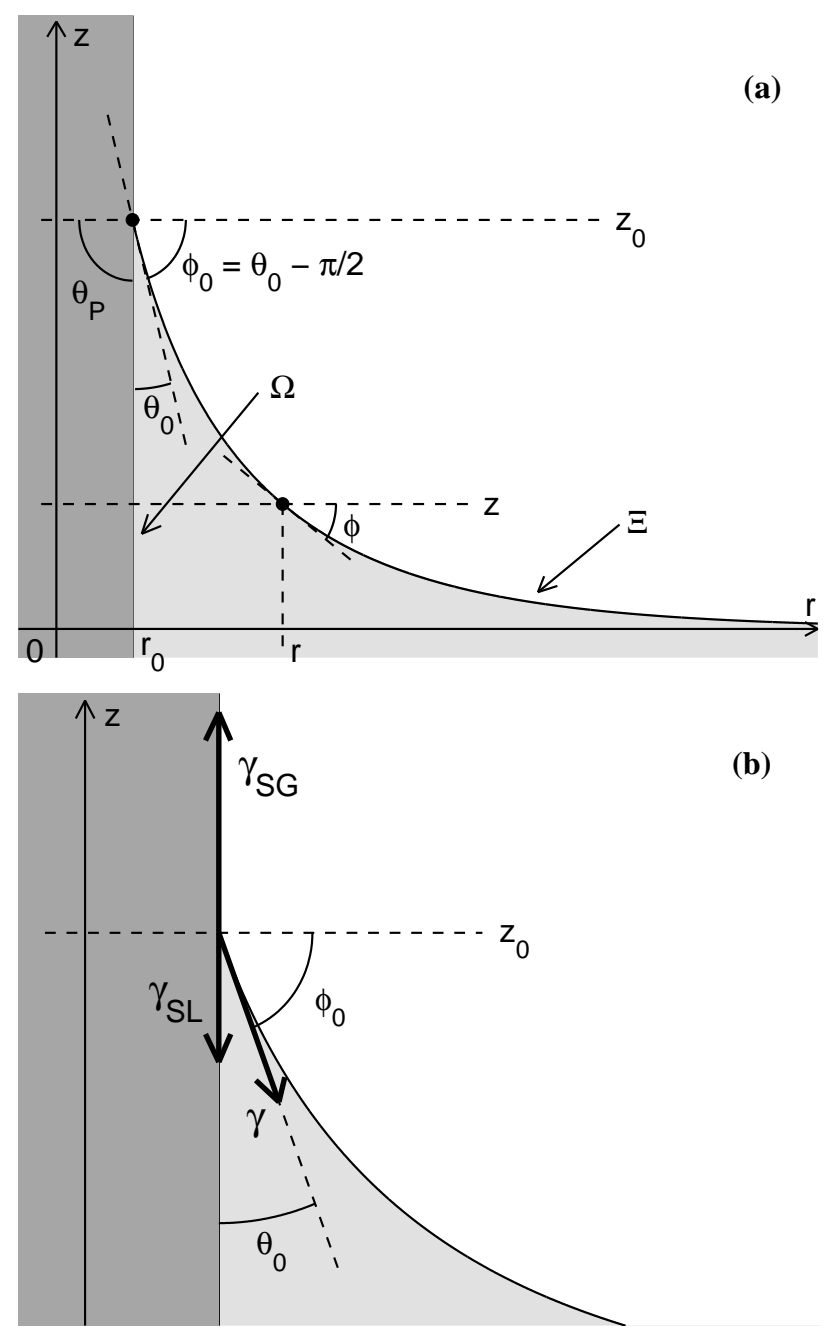

(b)

Figure 1: (a) A schematic demonstrating the wetting of a hydrophilic cylinder of constant circular cross-section. The solid is shown in dark grey, while the liquid phase is shown in light grey. The point $\left(r_{0}, z_{0}\right)$ indicates the threephase contact point(line). (b) Close-up view of the contact region. Arrows indicate the direction of surface tensions considered as directed forces per unit length. 
line of contact with the solid to give the boundary condition,

$$
\frac{\left.z_{r}\right|_{r=r_{0}}}{\sqrt{1+\left.z_{r}^{2}\right|_{r=r_{0}}}}=-\frac{\Delta \gamma}{\gamma}
$$

that the profile has to satisfy on the surface. Note that the slope at the cylinder is opposite in sign to $\Delta \gamma$, and since the profile is monotonic, the height of the contact line, $z_{0}$, will have the same sign as $\Delta \gamma$. Consequently, a hydrophilic cylinder $\Delta \gamma>0$ will see a meniscus rise, while a hydrophobic cylinder $\Delta \gamma<0$ will see a meniscus fall.

\subsection{Energy functional for ideal capillarity: infinite cylinder}

An application of the variational method begins with a definition of a functional, normally an energy functional that is to be minimized. In our case the functional is the energy change,

$$
\Delta F=\left(\gamma_{S L}-\gamma_{S G}\right) \Delta A_{\Omega}+\gamma \Delta A_{\Xi}+g\left(\rho_{L}-\rho_{G}\right) \int_{V \Xi} z d V,
$$

associated with the deformation of the fluid interface $\Xi$ and preferential wetting of the immersed solid. The first term represents the favorable increase in contact energy resulting in a change in contact area with the solid, $\Delta A_{\Omega}$. The second term represents the unfavorable increase in surface energy proportional to the increase in fluid interfacial area $\Delta A_{\Xi}$, resulting from the induced deformation of $\Xi$, propagating away from the solid. The last term is also an unfavorable contribution whose origin is the gravitational potential proportional to the mass of liquid displaced due to deformation. At equilibrium these combine in such a way that the total energy change is minimal. Although Equation (4) applies generally to the wetting problem, we confine attention here to a cylinder of infinite length and radius $r_{0}$, vertically immersed in a semi-infinite bath of liquid, with a gaseous phase filling the 
upper half-space. An axisymmetric meniscus is created with a three-phase circular contact line at height, $z_{0}$.

Suppose an arbitrary, axisymmetric wetting profile denoted by $\widetilde{z}(r)$ with meniscus height $z_{0}$ is created. The change in the energy of the system measured with respect to the undeformed interface (as reference state) can then be expressed as

$$
\Delta F=2 \pi \int_{r_{0}}^{\infty} f\left(r, \widetilde{z}, \widetilde{z}_{r}\right) d r-\Psi\left(z_{0}\right),
$$

where $f:=\gamma\left(r \sqrt{1+\widetilde{z}_{r}^{2}}-r\right)+g \Delta \rho r \widetilde{z}^{2} / 2$ is a sum of surface and gravitational contributions, associated with the second and third terms of (4). The first term of (4), the change in contact energy at the boundary, is here represented by $\Psi\left(z_{0}\right):=2 \pi \Delta \gamma r_{0} z_{0}$.

In the simplest variational approach one considers the set of possible profiles $(r, \widetilde{z}(r))$ that satisfy the end constraints of an asymptotic return to the undeformed state, $\widetilde{z} \rightarrow 0$ as $r \rightarrow \infty$, and a constant meniscus height at contact with the cylinder, $\tilde{z}\left(r_{0}\right)=z_{0}$. From this set one extracts the minimizing profile, denoted $z(r)$, by considering an arbitrary perturbed profile of the form $\widetilde{z}(r)=z(r)+\delta z(r) . \quad \delta z(r)$ is a suitably differentiable function that satisfies the same asymptotic constraint as $z$, but vanishes at $r=r_{0}, \delta z\left(r_{0}\right)=0$. These conditions ensure that the perturbed profiles have the correct height, $z_{0}$. A first-order approximation to the functional change, $\delta F[\delta z ; z]=\Delta F[z+\delta z]-\Delta F[z]$, is readily found following a substitution and expansion to first order in the perturbation, $\delta z$. By setting this first-order functional change to zero, $\delta F=0$, and invoking the DuboisReymond lemma $[2,6,7]$ one is lead to the usual Euler-Lagrange equation

$$
\frac{d}{d r} \frac{\partial f}{\partial z_{r}}-\frac{\partial f}{\partial z}=0
$$

Invoking the above expression for $f$ one regains Equation (1) to be satisfied by the minimizing profile, $z$. 

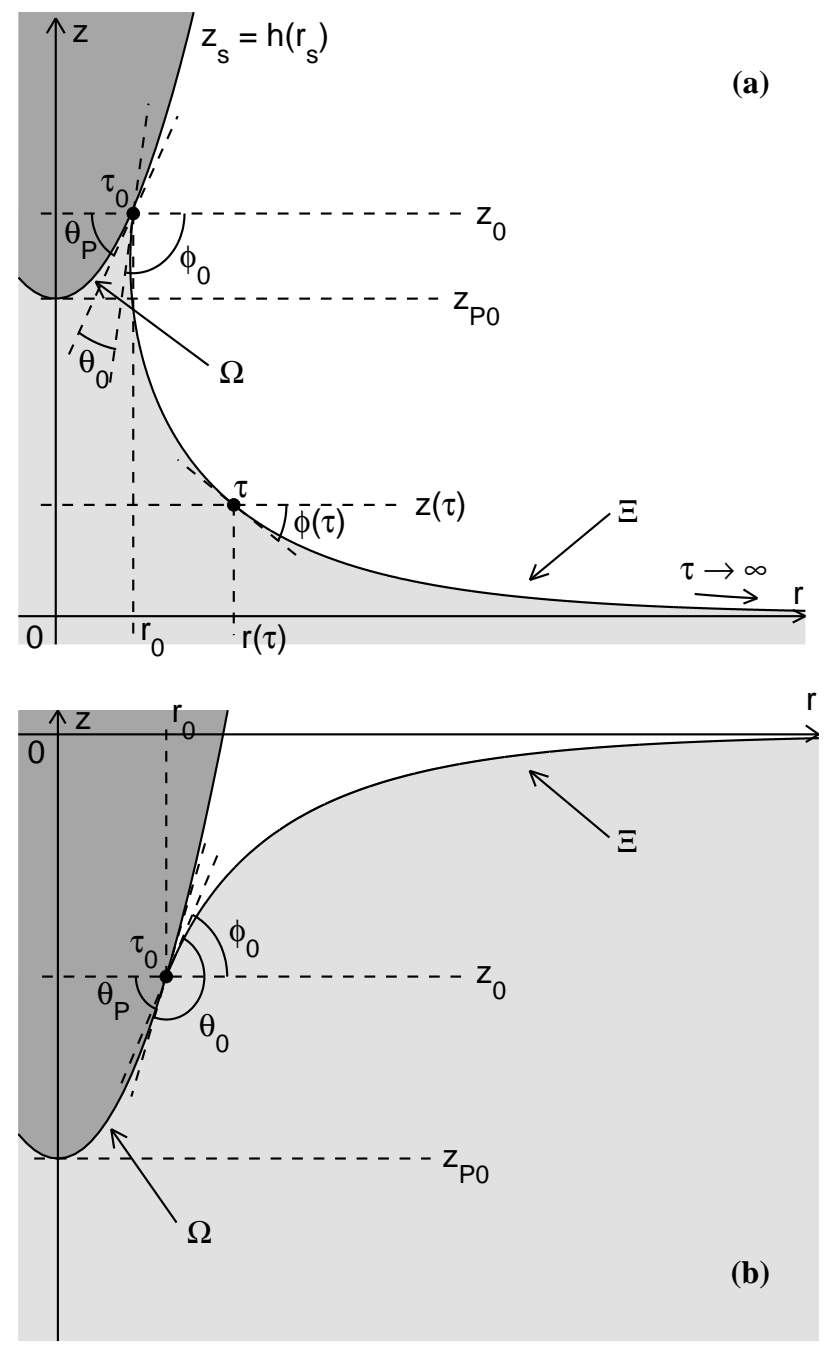

Figure 2: Cross-sectional schematics demonstrating the wetting of a semiinfinite paraboloid cylinder of shape, $z_{s}\left(r_{s}\right)=z_{p 0}+\lambda r_{s}^{2}$, being a special case of an axisymmetric solid of shape, $z_{s}\left(r_{s}\right)=h\left(r_{s}\right)$. Upper figure (a) shows a hydrophilic solid for which $z_{s}>0$. Lower figure (b) shows a hydrophobic solid that is partially immersed. 
To reproduce the boundary condition Equation (2) or equivalently (3), via the variational treatment is not typically demonstrated in undergraduate courses and requires somewhat more machinery. To avoid repetition and to save space, we take up this discussion in a more general context in the next section. The reader can then easily make the necessary association with the present special case.

In light of later discussions, we point out that what is often neglected or under emphasized in the teaching of the variational method: solving Equation (1) is a necessary but not sufficient condition of the minimizing profile. Solutions to (1) satisfying the boundary condition (2), may represent other forms of local extrema of the functional $\Delta F$. Moreover, without considering the second variation of $\Delta F$, one cannot generally distinguish between solutions that are local minima, maxima or saddle points, although an evaluation of the functional itself can give some insight.

\subsection{Energy functional for ideal capillarity: semi-infinite parabolic cylinder}

Consider now a similar system wherein the infinite cylinder is replaced by a semi-infinite, rotationally symmetric solid whose vertical cross-section is given by an injective, monotonic increasing function of the form $z_{s}=h\left(r_{s}\right)$; $d h / d r_{s}=: h_{r_{s}} \geq 0$. As in the previous section, a wetting meniscus is created when the solid is partially immersed in the liquid bath. The circular, threephase contact line again takes on a height, $z_{0}$. Note, that the finite slope of the solid at the contact line, $\left.h_{r_{s}}\right|_{r_{0}}$, opens up the possibility that the deformed gas-liquid interface, $\Xi$, is a non-injective function of $r$. Consequently, a parametric representation of the profile, $(r(\tau), z(\tau))$, is better suited. Here, we choose to use as parameter the arclength variable, $\tau$, measured along the vertical cross-sectional profile (see Figure 2). The energy functional of the 
system is then

$$
\Delta F=\int_{0}^{\infty} f\left(\widetilde{r}, \widetilde{z}, \widetilde{r}_{\tau}, \widetilde{z}_{\tau}\right) d \tau-2 \pi \gamma \int_{0}^{\infty} r d r-\Psi\left(r_{0}, z_{0}\right),
$$

where

$$
f:=2 \pi \gamma \widetilde{r} \sqrt{\widetilde{r}_{\tau}^{2}+\widetilde{z}_{\tau}^{2}}-\pi g \Delta \rho \widetilde{r}^{2} \widetilde{z} \widetilde{z}_{\tau}
$$

and

$$
\Psi(r, z):=\frac{\pi}{2} g \Delta \rho \widetilde{r}^{2} \widetilde{z}^{2}+2 \pi \int_{0}^{r}\left(\Delta \gamma r_{s} \sqrt{1+h_{r_{s}}^{2}}-g \Delta \rho r_{s} \frac{h\left(r_{s}\right)^{2}}{2}\right) d r_{s} .
$$

As in Section 2.2, implementing the variational method involves adopting optimal profile functions, $r(\tau)$ and $z(\tau)$, and expressing an arbitrary profile by the pair $(\widetilde{r}, \widetilde{z})$ where $\widetilde{r}(\tau)=r(\tau)+\delta r(\tau)$ and $\widetilde{z}(\tau)=z(\tau)+\delta z(\tau)$. As before, the perturbations $\delta r$ and $\delta z$ will satisfy the asymptotic conditions $\delta r, \delta z \rightarrow 0$ as $\tau \rightarrow \infty$. On the other hand, at the object-end of the profile we set no requirements on the perturbation values, bar one. A natural inherent constraint is that whatever form the surface profile eventually takes, it must reside on or be connected to the solid surface, $S\left(r_{s}, z_{s}\right)=h\left(r_{s}\right)-z_{s}=0$. Consequently, both pairs $(\widetilde{r}, \widetilde{z})$ and $(r, z)$ must adhere to this constraint, which then prevents the perturbations $(\delta r, \delta z)$ from varying completely independently. This additional consideration can be included in a number of ways [2], most simply by creating a modified functional

$$
\mathbb{F}=\Delta F+\mu S,
$$

where $\mu$ is a Lagrange multiplier. Variation of $\mathbb{F}$ can now be effected without consideration of the constraint. The first-order approximation to the functional change, $\delta \mathbb{F}[\delta r, \delta z ; r, z]=\mathbb{F}[r+\delta r, z+\delta z]-\mathbb{F}[r, z]$, found by expanding to first order in the perturbations, $\delta r$ and $\delta z$, which are now completely independent, possesses several contributions. First there is the pair of Euler-Lagrange differential equations

$$
\frac{d}{d \tau} \frac{\partial f}{\partial r_{\tau}}-\frac{\partial f}{\partial r}=0, \quad \frac{d}{d \tau} \frac{\partial f}{\partial z_{\tau}}-\frac{\partial f}{\partial z}=0
$$


arising from terms in the integral, $\Delta F$, that are proportional to $\delta r$ and $\delta z$, respectively. Then there arise expressions proportional to the perturbations evaluated at the boundary, $\delta r(\tau=0)$ and $\delta z(\tau=0)$, respectively,

$$
\left.\mu \frac{\partial S}{\partial r}\right|_{0}-\left.\left(\frac{\partial f}{\partial r_{\tau}}+\frac{\partial \Psi}{\partial r}\right)\right|_{0}=0,\left.\quad \mu \frac{\partial S}{\partial z}\right|_{0}-\left.\left(\frac{\partial f}{\partial z_{\tau}}+\frac{\partial \Psi}{\partial z}\right)\right|_{0}=0 .
$$

Eliminating the Lagrange multiplier from between these two equations gives what Courant and Hilbert [2] refer to as a natural boundary condition

$$
\left.\left[\left(\frac{\partial f}{\partial r_{\tau}}+\frac{\partial \Psi}{\partial r}\right) \frac{\partial S}{\partial z}\right]\right|_{0}=\left.\left[\left(\frac{\partial f}{\partial z_{\tau}}+\frac{\partial \Psi}{\partial z}\right) \frac{\partial S}{\partial r}\right]\right|_{0}
$$

corresponding to the requirement that the solution be connected to the solid surface.

Introducing the signed angle $\phi(\tau)$ between the horizontal and the tangent to the profile at the point $(r(\tau), z(\tau))$ (see Figure 2a), and inserting the function $f$ from (6), the two second-order Euler-Lagrange differential equations can be rewritten more conveniently as a set of three first-order differential equations

$$
r_{\tau}=\cos \phi(\tau), \quad z_{\tau}=\sin \phi(\tau), \quad \phi_{\tau}=\frac{g \Delta \rho}{\gamma} z(\tau)-\frac{\sin \phi(\tau)}{r(\tau)} .
$$

This system is the parametric analogue of the Young-Laplace equation (1).

With $f, \Psi$ and $S$ explicitly implemented in (8), the boundary equation becomes

$$
\frac{\left(\left.z_{\tau}\right|_{0}\right)\left(\left.h_{r}\right|_{r_{0}}\right)+\left.r_{\tau}\right|_{0}}{\sqrt{\left(\left.r_{\tau}\right|_{0}\right)^{2}+\left(\left.z_{\tau}\right|_{0}\right)^{2}} \sqrt{1+\left(\left.h_{r}\right|_{r_{0}}\right)^{2}}}=-\frac{\Delta \gamma}{\gamma}
$$

which is independent of gravitational properties. This general boundary condition must be satisfied by solutions to (9), and it follows from a direct application of the natural boundary conditions of variational calculus. Contrast (10) with its counterpart in the right circular cylinder case, (3). Extracting the factor $\left|r_{\tau}\right|_{0} \mid$ and taking the limit $|d h / d r| \rightarrow \infty$ at $r=r(\tau=0)=r_{0}$ 


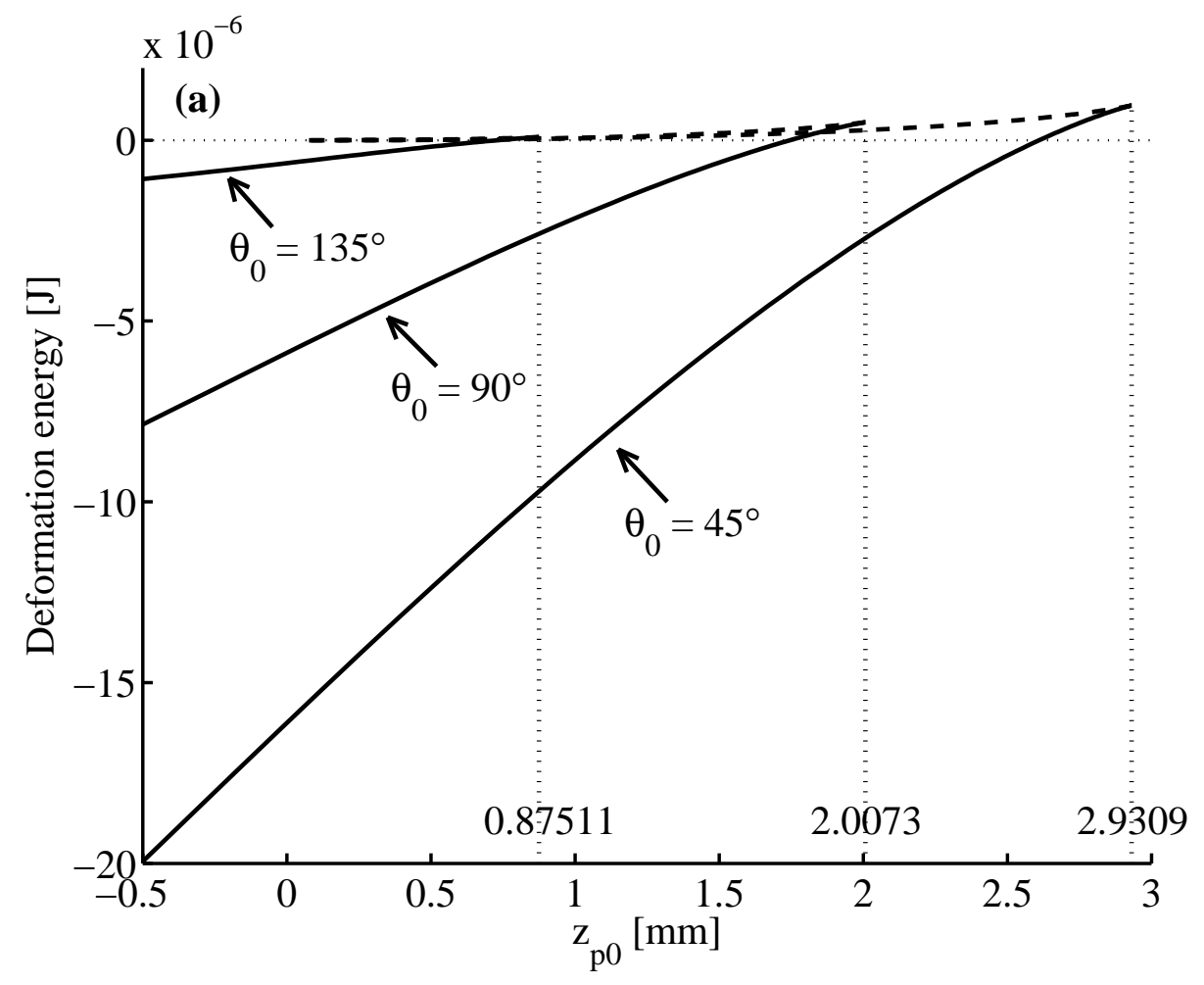

Figure 3: The change in free energy, $\Delta F$, due to deformation as a function of solid height, $z_{p 0}$, for the stable (solid lines) and unstable (dashed lines) solutions, for three values of the contact angle, $\theta_{0}$. The respective existence limits, $z_{p 0, \max }$, indicated in the figure are given in $\mathrm{mm}$. Physical parameters are: $\Delta \rho=996.91 \mathrm{~kg} / \mathrm{m}^{3}, \gamma=0.0728 \mathrm{~N} / \mathrm{m}, \lambda=50 \mathrm{~m}^{-1}, \theta_{0}=35^{\circ}, 90^{\circ}$ and $135^{\circ}$. 
reduces (10) to (3). Upon substituting $\cos \phi_{0}$ and $\sin \phi_{0}$ for the profile derivatives, $r_{\tau}$ and $z_{\tau}$, and $\tan \theta_{P}$ for $\left.h_{r}\right|_{r_{0}}$ in (10), we obtain

$$
\sin \theta_{P} \sin \phi_{0}+\cos \theta_{P} \cos \phi_{0} \equiv \cos \left(\theta_{P}-\phi_{0}\right)=-\frac{\Delta \gamma}{\gamma},
$$

which one easily recognizes as the Young-Dupre relation (2) for the contact angle $\theta_{0}=\phi_{0}-\theta_{P}+\pi$. Despite the fundamental geometrical difference to the cylinder system, we have retrieved the same physical boundary condition, independent of both gravitational and geometric properties. Yet, the shape of the solid still matters for determining the initial angle, $\phi_{0}$, via the factor $\left.h_{r}\right|_{r_{0}}$ in (10).

\section{Numerical results for a semi-infinite paraboloid}

For a numerical study of the wetting problem we chose the specific case of a semi-infinite paraboloid of vertical cross-section, $z\left(r_{p}\right)=z_{p 0}+\lambda r_{p}^{2}$. Prosaically, this is a solid in the shape of a pin, with apex at height $z_{p 0}$, and splay measured by the parameter $\lambda$. The numerical task involves solving the system of differential equations (9) with boundary condition (10). The three a priori unknown boundary values, $r_{0}, z_{0}$ and $\phi_{0}$, needed to specify a profile, are interrelated: $r_{0}$ and $z_{0}$ via the solid geometry; $\phi_{0}=\theta_{0}+\theta_{P}-\pi$ with $\theta_{0}$ given by the Young-Dupre relation (2); and $\theta_{P}$ by the slope of the solid:

$$
\phi_{0}=\theta_{P}+\theta_{0}-\pi=\tan ^{-1}\left(\left.h_{r}\right|_{r_{0}}\right)+\cos ^{-1}\left(\frac{\Delta \gamma}{\gamma}\right)-\pi .
$$

The remaining unknown is determined iteratively by matching an asymptotic approximation at a finite radius $r_{\infty}=r\left(\tau_{\infty}\right)$

$$
z(r)=C K_{0}(\eta r)
$$

where $\eta=(g \Delta \rho / \gamma)^{1 / 2}$. A combined Newton-Raphson and Runge-Kutta iteration method is used; details are sketched out in the algorithm below. 

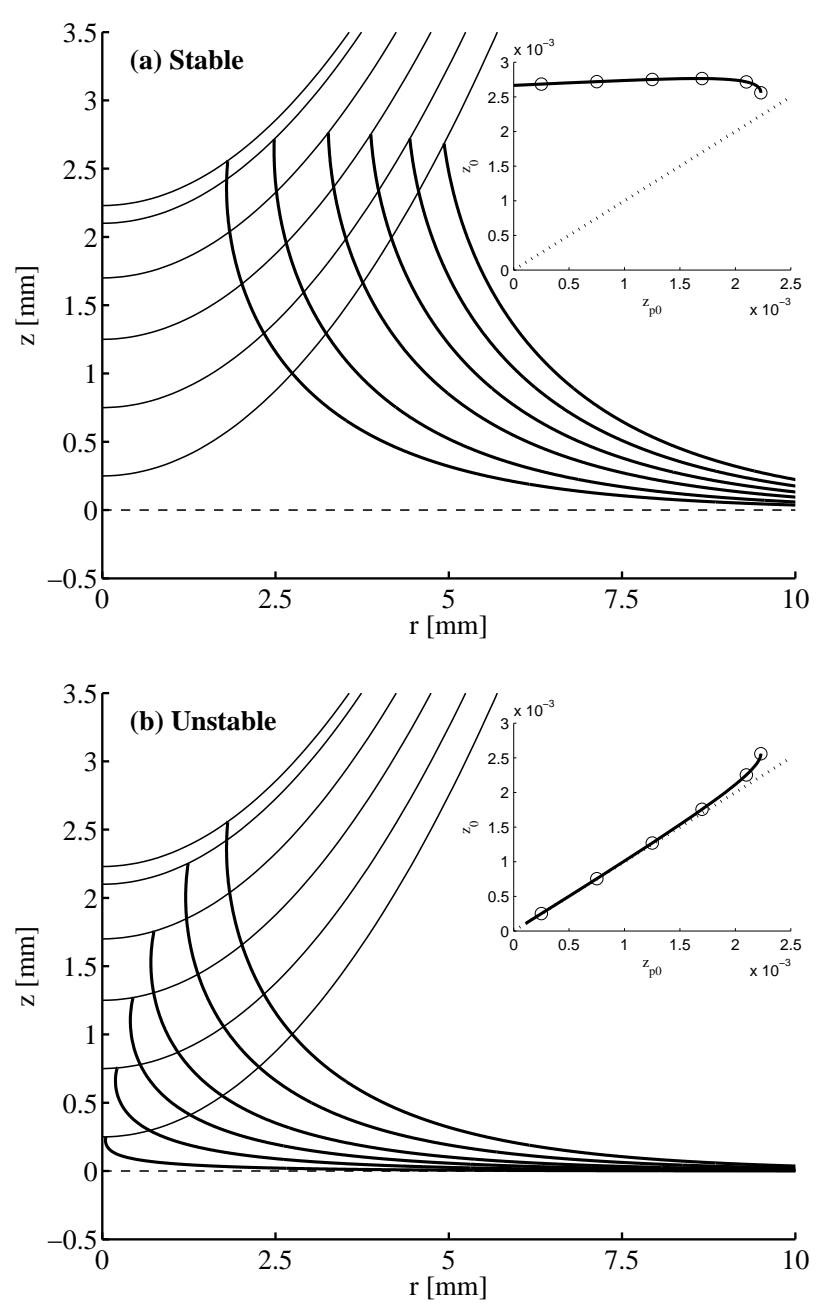

Figure 4: Stable (a) and unstable (b) solutions of (9) (thick lines) and their behavior as a function of height of paraboloid (thin lines). The wetting profile common to both figures corresponds to the critical solution, at the maximum paraboloid height, $z_{p 0 \text {, max }}=2229.994 \mu \mathrm{m}$. The insets represent the contact line height (solid lines) as a function of paraboloid height (dotted lines). Physical parameters are: $\Delta \rho=996.91 \mathrm{~kg} / \mathrm{m}^{3}, \gamma=0.0728 \mathrm{~N} / \mathrm{m}, \theta_{0}=$ $60^{\circ}, \lambda=100 \mathrm{~m}^{-1}$. 


\section{Algorithm}

- fix $r_{\infty}$ (along with all other physical parameters); guess $z_{\infty}$; then deduce the following

$$
C=\frac{z_{\infty}}{K_{0}\left(\eta \cdot r_{\infty}\right)}, \quad z_{r}\left(r_{\infty}\right)=-C \cdot \eta \cdot K_{1}\left(\eta \cdot r_{\infty}\right), \quad \phi_{\infty}=\tan ^{-1}\left(z_{r}\left(r_{\infty}\right)\right) .
$$

- solve the differential equations (9) backwards (to maintain numerical stability, $\tau$ goes from $\tau_{\infty}$ to 0$)$ until the profile crosses or misses the paraboloid. This gives $\left\{r_{0}, z_{0}, \phi_{0}\right\}$.

- using $\left\{r_{0}, z_{0}\right\}$, obtain the corresponding theoretical angle $\phi_{0, \text { theo via }}(10)$.

- compare the numerically obtained $\phi_{0}$ with the theoretical angle $\phi_{0, \text { theo }}$ and correct the guess $z_{\infty}$ accordingly.

\subsection{Stable and unstable profiles}

For a given set of physical parameters, the above simple numerical method gives, in general, a pair of solutions, both reasonable looking gas-liquid interfaces. However, physics dictates that they cannot both be local minima of the energy functional. The possibility and significance of finding more than one solution to the governing equations should be kept in mind. This possibility is inherent in this system of nonlinear equations, and is the second appealing feature of this example. Solutions of system (9) satisfying boundary condition (10), are stationary points of the energy $\Delta F$, and are thus either local minima, maxima or saddle points of the energy surface. Although the second variation of (5) should ultimately be considered to distinguish between the types of extrema, distinction can be achieved by means of simple physical arguments when the number of solutions is small.

Since the two solutions are successive local extrema, one must be a local maximum (or a saddle point) of $\Delta F$, and hence an unstable solution. This 
is supported by the fact that the energy change (5) for one of the solutions is systematically larger than for the other. Figure 3 shows the two branch lines representing the stable and unstable energies. If plotted in $3 \mathrm{D}$ the stable branch would trace out an energy valley, while the unstable branch would be trace out a ridge on the energy surface.

The behavior of the two solutions as a function of the paraboloid height parameter, $z_{p 0}$, is illustrated in Figure 4 . The profile corresponding to the lowest energy, nominally the stable solution, exhibits physically expected behavior. As the solid is immersed in the liquid, the air-water interface is shifted aside and the radius of the contact line, $r_{0}$, increases steadily (Figure 4a). The second solution exhibits very different and very unphysical behavior (Figure 4b). As the solid is lowered, the unstable profile slides in under it, reducing both the gravitational and surface contributions to the energy. However, the contact area with the solid, $\Delta A_{\Omega}$, is also reduced as is then the favorable contact energy. The end result is a higher energy than is attributed to the stable profile; hence the reference to an unstable solution. Note that since the unstable profile squeezes itself in between the solid and the reference level, $z=0$, it exists only for positive paraboloid heights $z_{p 0}$. The stable solution, on the other hand exists for any, $z_{p 0}$, negative or positive (the latter up to a point).

Naturally, the stable solution is the only one selected by nature and as such is the only reproducible profile, and the only one of physical interest.

\subsection{Stability limit}

An understanding of both the physical system and the variational method, we have found, can be enhanced by the existence of more than one solution. Tracking both stable and unstable solutions as a function of increasing $z_{p 0}$, shows that both stable and unstable profiles tend to converge, eventually merging at a certain value, $z_{p 0 \text {,max }}$, of the solid height. From Figure 4 , as $z_{p 0}$ 
increases the stable profiles approach the coincident profile from the right, with contact radius, $r_{0}$, decreasing, while the unstable profiles approach from the left, with both $r_{0}$ and $z_{0}$ steadily increasing. The key feature of this convergence is that for solid heights above $z_{p 0 \text {,max }}$ there are no solutions to (9) satisfying (10), stable or unstable. $z_{p 0 \text {,max }}$ therefore characterizes the absolute stability limit of the system. Moreover, the convergence of the two solutions to a unique critical profile provides an effective numerical method to determine the well-defined existence limit of physical solutions. The physical significance of this is that there is a practical limit to which an object can be raised while still maintaining contact with a wetting meniscus.

Figure 5a shows the contact line radius $r_{0}$ as a function of $z_{p 0}$, for solids having four different values of contact angle $\theta_{0}$. The contact radii of the stable and unstable profiles monotonically decrease and increase, respectively, as $z_{p 0}$ increases, to merge at the limiting height $z_{p 0 \text {,max }}$. As the paraboloid is immersed (that is, $z_{p 0}$ decreases) the three-phase contact region becomes increasingly broad and the contact radii for the stable profiles diverge (solid lines, left side of the figure). In contrast, the unstable contact radii approach 0 as $z_{p 0}$ decreases arriving at this limit when $z_{p 0}=0$; the profiles cease to exist for negative $z_{p 0}$. Figure $5 \mathrm{~b}$ and the insets in Figure 4, show the dependence of the meniscus height $z_{0}$ on $z_{p 0}$, for the same four values of $\theta_{0}$. While the unstable meniscus height grows monotonically with $z_{p 0}$, the stable meniscus height $z_{0}$ reaches a maximum (indicated by a square symbol on the solid curves in Figure 5b) before decreasing until it meets with its unstable counterpart at the limit $z_{p 0 \text { max }}$. Finally, Figure $5 \mathrm{c}$ shows the $z_{p 0}$-dependence of the angle that the meniscus at contact makes with the horizontal, $\phi_{0}$, for the same four values of $\theta_{0}$. As with the other properties, both the stable and unstable meniscus angles converge at a stability limit as the solid is raised. At the other extreme, $z_{p 0} \rightarrow-\infty$, the inner angle the solid makes with the horizontal approaches $\pi / 2$ and the stable values of $\phi_{0}$ then converge to $\theta_{0}-\pi / 2$, as in the case of the cylinder. 

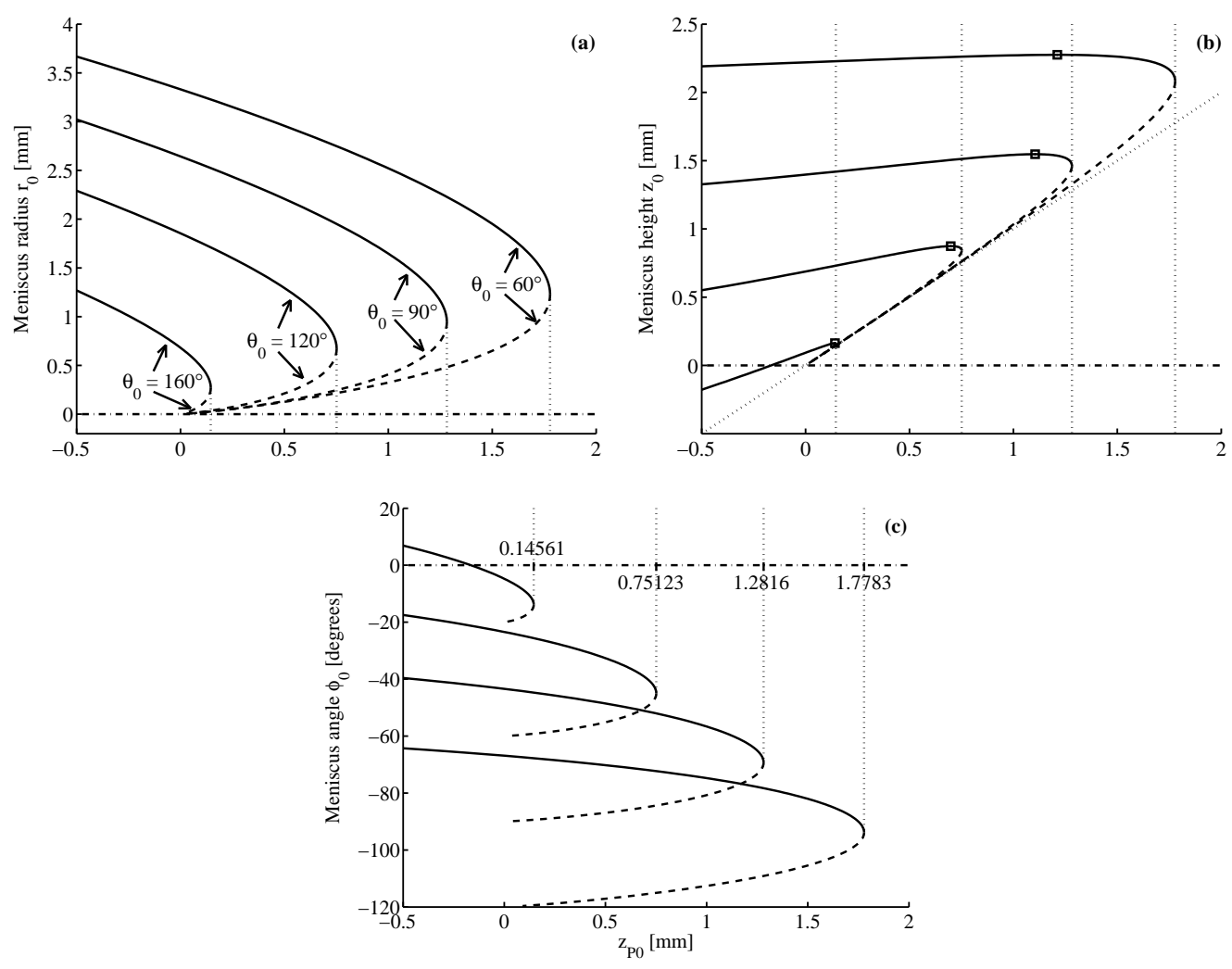

Figure 5: The meniscus coordinates. (a) $r_{0}$, (b) $z_{0}$, and (c) $\phi_{0}$ as functions of paraboloid height, $z_{p 0}$, for the stable (solid lines) and unstable (dashed lines) solutions, for four values of contact angle, $\theta_{0}$. The vertical dotted lines indicate the different existence limits, $z_{p 0 \text {,max }}$, with the numerical values given in (c) in $\mathrm{mm}$. The diagonal dotted line in (b) represents the paraboloid height. Physical parameters are: $\Delta \rho=996.91 \mathrm{~kg} / \mathrm{m}^{3}, \gamma=0.0728 \mathrm{~N} / \mathrm{m}, \lambda=$ $200 \mathrm{~m}^{-1}, \theta_{0}=60^{\circ}, 90^{\circ}, 120^{\circ}$ and $160^{\circ}$. The individual contact angle values are indicated in (a). 


\section{Summary remarks}

The pedagogic benefit of a good physical example to illustrate abstract mathematical notions should not be underestimated. Particularly worthwhile is an example that addresses several different aspects. The problem we have described of determining the equilibrium shape of a wetting meniscus adjacent to a solid of simple geometry is rich in features that help clarify the variational method. It illustrates how the more abstract variational boundary equations can be implemented. It also represents a system that gives rise to both minimum and maximum of the energy, an occurrence not normally taken up in standard texts. Furthermore, by utilizing these two solutions it is possible to establish a criteria that earmarks eventual existence limits of solutions of the variational equations. The proposal has the further appeal that it is conceptually simple in terms of addressing a common-place phenomena whose behavior is physically tangible to undergraduate students.

The only negative aspect of this proposal is that solutions to the variational equations must be obtained numerically. This might restrict the teacher to raise this example in ready made form as part of lecture material, or to use it in a problem based learning context. However, solutions to the governing equations can be obtained readily using standard differential equation solvers in commercial software packages such as Matlab.

\section{References}

[1] P. M. Morse and H. Feschback, Methods of Theoretical Physics (McGraw-Hill, New York, 1952). C895

[2] R. Courant and D. Hilbert, Methods of Mathematical Physics (Wiley, New York, 1965), Vol. 1. C895, C896, C901, C904, C905 
[3] S. Symon, Mechanics, 3rd ed. (Addison-Wesley, Reading, MA, 1971). C895

[4] H. Goldstein, Classical Mechanics, 2nd ed. (Addison-Wesley, Singapore, 1980). C895

[5] G. B. Arfken and H. J. Weber, Mathematical Methods for Physicists, 5 ed. (Academic Press, New York, 2001). C895

[6] G. M. Ewing, Calculus of Variations with Applications (Dover. Original publication Norton \& Co, 1969, New York, 1985). C895, C901

[7] M. Renardy and R. C. Rogers, An Introduction to Partial Differential Equations (Springer Verlag, New York, 1992). C895, C901

[8] F. P.-A. Cortat and S. J. Miklavcic, Phys. Rev. E 68, 052601 (2003). C896

[9] F. P.-A. Cortat and S. J. Miklavcic, Langmuir 20, 3208 (2004). C896

[10] A. W. Neumann and J. K. Spelt, Applied Surface Thermodynamics (Marcel Dekker Inc., 1996). C897

[11] G. K. Batchelor, Introduction to Fluid Dynamics (Cambridge University Press, Cambridge, 1967). C898 